\title{
Advanced Linear Processing for 2-Hop Fixed MIMO Relaying
}

\author{
Pei Xiao, Mathini Sellathurai, Colin Cowan \\ The Institute of Electronics, Communications and Information Technology \\ Queen's University Belfast, BT3 9DT, United Kingdom \\ E-mail: \{pei.xiao,m.sellathurai,c.cowan\}eecit.qub.ac.uk
}

\begin{abstract}
In this paper, we consider a 2-hop downlink pointto-multipoint fixed relay systems and propose a novel linear processing strategy at the base station and relay station to improve the reliability of the source-relay and relay-to-destination links. When coupled with a soft-decode-and-forward protocol, the proposed scheme leads to significant performance gain compared to the conventionl solutions for fixed relay networks.
\end{abstract}

\section{INTRODUCTION}

Wireless communication networks allow user terminals or mobile stations (users) to move within the network without losing the ability to continue communication. However, each station has limited coverage due to the limitation on signal strength and the fact that the signal strength degrades over distance. As the distance between users and station increases, the strength of the signal received becomes lower and the transmission quality becomes lower. In addition, signal quality and coverage may be affected by factors such as physical structures, signal interference, weather, transmission conditions, formats, etc.. Therefore, coverage gaps or holes may exist and users in those areas may have limited or no network access.

One solution to avoid or reduce coverage holes is to provide more base stations to enhance the communication coverage and system capacity. However, system cost increases as the number of station increases. A better alternative is to use fixed relay [1]. Since it implements a subset of base station functions, fixed relay is a low cost and low complexity solution to boost coverage and link capacity in cellular networks when carefully placed at the cell edge or in regions with significant shadowing. The cooperative relaying technology is considered as a potential candidate by the 3GPP LTE (Long Term Evolution) standard to improve e.g., the coverage of high data rates, group mobility, temporary network deployment, the cell-edge throughput and/or to provide coverage in new areas.

The existing cooperative diversity protocols fall into two categories: i) repetition-based cooperative diversity protocol, such as amplify-and-forward (AF), decode-and-forward (DF), selection relaying, incremental relaying [2], [3]; ii) space-timecoded cooperative diversity protocol [4], [5]. In a cellular system, there are usually a large number of users to be served in one cell, high capacity gains can be achieved by transmitting multiple streams to different users sharing the same time-frequency resources. This technique is known as Multi-User MIMO (MU-MIMO) [6]. Most previous work in the area of cooperative relay mainly focuses on point-to-point transmission via relays [2]-[5], [7]. In practical systems, only a few fixed relays are available in each cell, consequently, each fixed relay will need to support multiple users. This motivates point-to-multipoint relaying solutions [8]-[10], where the relay forwards data to and from multiple users. This paper focuses on the point-to-multipoint fixed relay, which has received less attention compared to the point-to-point solution. The main challenge is to provide a high-capacity link between the base station and relay, while at the same time provide multiple data links to multiple users. We propose a soft-decode-andforward (SDF) protocol and advanced multiuser MIMO linear precoding schemes to meet this challenge.

The remainder of the paper is organized as follows. The downlink multiuser MIMO relaying system is introduced in Section II. The proposed SDF protocol and precoding scheme are described in Section III. The effectiveness of the proposed schemes are demonstrated in Section IV with computer simulations, and conclusions are drawn in Section V.

Throughout this paper, $(\cdot)^{T}$ denotes matrix transpose, $(\cdot)^{H}$ matrix conjugate transpose, $(\cdot)^{*}$ matrix conjugate, $\mathrm{E}[\cdot]$ expectation, trace $(\cdot)$ trace operation, and $\mathbf{I}_{N}$ an $N \times N$ identity matrix.

\section{System MOdeL}

The proposed two-hop relay system is depicted in Fig. 1. In the first time slot the relay receives and stores the signal from the base station (BS), and detects and applies a linear filter, then forwards the processed signal to multiple users creating a multiuser MIMO relay in the second time slot. As such, a high-throughput MIMO link can be employed between the BS and fixed relay, the MIMO broadcast channel (BC) can be used to deliver data to/from multiple users. Just as previous two-hop works [8]-[11], we also neglect the direct connection between the BS and users. This simplifies analysis and is realistic for the case when the mobile is near the cell edge or is highly shadowed.

We assume the BS has $N_{b}$ transmit antennas, the relay station (RS) has $N_{r}$ transmit and receive antennas and $K$ mobile users each has one antenna. At the BS, the symbol vector $\mathbf{s} \in \mathbb{C}^{B \times 1}$ is precoded using the precoding matrix $\mathbf{P}_{1} \in \mathbb{C}^{N_{b} \times B}$, i.e., $\mathbf{x}=\mathbf{P}_{1} \mathbf{s} \in \mathbb{C}^{N_{b} \times 1}$, where the symbol vector $\mathbf{s}=\left[\begin{array}{lll}s_{0} & \ldots & s_{B}\end{array}\right]^{T}$ comprises the transmit symbol of $B$ parallel data streams. The received signal vector $\mathbf{y}_{r} \in$ 
Base Station (BS)

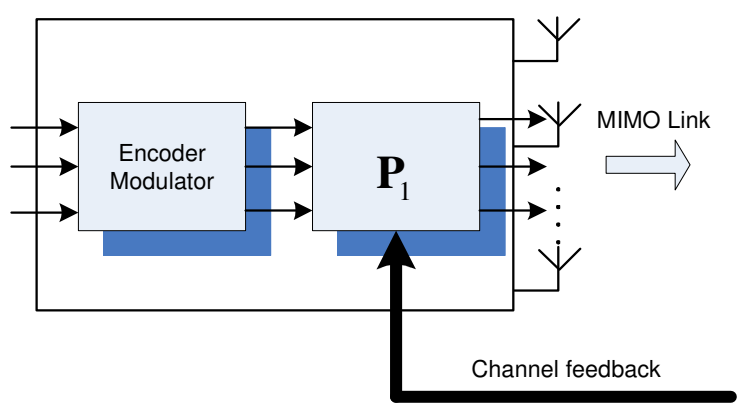

Fig. 1. Block diagram of the proposed 2-hop MIMO relaying system.

$\mathbb{C}^{N_{r} \times 1}$ at the relay station can be expressed as

$$
\begin{aligned}
\mathbf{y}_{r} & =\beta_{1}^{-1}\left(\mathbf{H}_{1} \mathbf{P}_{1} \mathbf{s}+\mathbf{n}_{1}\right)=\beta_{1}^{-1}\left(\mathbf{H}_{1} \beta_{1} \tilde{\mathbf{P}}_{1} \mathbf{s}+\mathbf{n}_{1}\right) \\
& =\mathbf{H}_{1} \tilde{\mathbf{P}}_{1} \mathbf{s}+\mathbf{n}_{1} / \beta_{1},
\end{aligned}
$$

where $\mathbf{H}_{1} \in \mathbb{C}^{N_{r} \times N_{b}}$ denotes the MIMO channel between the BS and the RS, $\tilde{\mathbf{P}}_{1}=\mathbf{P}_{1} / \beta_{1}$, and $\mathbf{n}_{1} \in \mathbb{C}^{N_{r} \times 1}$ is the white Gaussian noise vector with zero mean and covariance matrix $\sigma_{n_{1}}^{2} \mathbf{I}_{N_{r}}$. The power normalization factor $\beta_{1}$ can be derived based on the power constraint $\left\|\mathbf{P}_{1} \mathbf{s}\right\|^{2}=\operatorname{trace}\left(\mathbf{P}_{1} \mathbf{P}_{1}^{H}\right)=$ $\operatorname{trace}\left(\beta_{1}^{2} \tilde{\mathbf{P}}_{1} \tilde{\mathbf{P}}_{1}^{H}\right)=N_{b} \sigma_{s}^{2}$ (where $\sigma_{s}^{2}$ is the average symbol energy), resulting in

$$
\beta_{1}=\sqrt{\frac{N_{b} \sigma_{s}^{2}}{\operatorname{trace}\left(\tilde{\mathbf{P}}_{1} \tilde{\mathbf{P}}_{1}^{H}\right)}} .
$$

Two basic strategies in cooperative relay systems are decode-and-forward (DF) and amplify-and-forward (AF). With the DF protocol, the relay station detects the received signal and performs linear processing on the reconstructed signal, then forward the processed signal to multiple users. The received signal at mobile stations (MSs) can be written as

$$
\mathbf{y}_{d}^{D F}=\beta_{2}^{-1}\left(\mathbf{H}_{2} \mathbf{P}_{2} \tilde{\mathbf{s}}+\mathbf{n}_{2}\right),
$$

where $\tilde{\mathbf{s}}$ is the reconstructed transmit signal vector at the relay station, $\mathbf{H}_{2} \in \mathbb{C}^{K \times N_{r}}$ denotes the MIMO channel between the RS and $K \mathrm{MSs}, \mathbf{P}_{2}$ is the RS precoding matrix, and $\mathbf{n}_{2} \in$ $\mathbb{C}^{K \times 1}$ is the white Gaussian noise vector with zero mean and covariance matrix $\sigma_{n_{2}}^{2} \mathbf{I}_{K}$. The power normalization factor $\beta_{2}$ can be derived similarly as (2).

In an AF based relay system, the RS amplifies the received signal without any attempt to decode, and forwards the amplified signal. The received signal at the destination node can be written as

$$
\begin{aligned}
\mathbf{y}_{d}^{A F} & =\beta_{2}^{-1}\left(\mathbf{H}_{2} \mathbf{P}_{2} \mathbf{y}_{r}+\mathbf{n}_{2}\right) \\
& =\beta_{2}^{-1} \beta_{1}^{-1} \mathbf{H}_{2} \mathbf{P}_{2} \mathbf{H}_{1} \mathbf{P}_{1} \mathbf{s}+\beta_{2}^{-1} \beta_{1}^{-1} \mathbf{H}_{2} \mathbf{P}_{2} \mathbf{n}_{1}+\beta_{2}^{-1} \mathbf{n}_{2} .
\end{aligned}
$$

We focus on the DF based relay system in this work, the AF based system expressed above is only used for comparison purposes.

\section{SOFT DECODE-AND-FORWARD PROTOCOL}

The DF protocol benefits from error correction probability and can fully or partly correct errors during the sourcerelay transmission. However, this is highly dependent on the reliable source-relay link otherwise it will lead to serious error propagation as relay will forward erroneous data and will mislead the destination receiver. In contrast, the AF protocol keeps itself from any premature decision and in fact preserves the soft information content of the received signal. However, this scheme fails to benefit from error correction possibility at relay and also amplifies and forwards the noise. Compared to $\mathrm{DF}, \mathrm{AF}$ is less complex. For this reason, some of previous works in fixed relay networks only consider AF approach for simplicity [10], [11]. However, we will mainly consider the DF protocol in order to robustify the link reliability and improve the system performance. In this context, the use of the DF protocol is justified by the fact that the additional complexity (compared to the AF protocol) is only spent at the RS, rather than in the MSs, and RS has much larger signal processing capacities than MSs.

As mentioned previously, one issue to be addressed is the error propagation problem associated with the conventional DF scheme. This problem is more severe when the source-relay link is in outage. Inspired by soft information preservation property of AF, a modified DF scheme, called soft DF was proposed in [12], [13] to construct the forward signal from soft values of the transmitted bits. The soft DF maintains the soft information content throughout the whole decoding and re-encoding process, and is shown to outperform both AF and DF schemes and inherits merits of both. The soft DF protocol presented in [12], [13] was designed for point-to-point relays. Here, we extend it to the point-to-multipoint relaying system under consideration.

In the downlink scenario, assuming that the transmitter has access to the channel state information (CSI) of all users, joint processing (precoding) can be performed at the transmitter (BS or RS). Precoding algorithms for multiuser MIMO can be categorized into linear and nonlinear schemes. The former achieves reasonable throughput performance with lower complexity; whereas the latter scheme provides near 
optimal solution at the expense of much higher complexity. From the implementation prospective, the linear approach is more feasible for practical implementation, and is therefore considered for real wireless communications systems, such as WiMAX and LTE. In what follows, we show how the soft DF system can be designed with the conventional and an improved linear precoding schemes.

\section{A. soft DF protocol with conventional precoding}

To facilitate the algorithm development, we derive the soft DF scheme based on the zero-forcing $(\mathrm{ZF})$ based linear precoders which completely decouple the signals from different transmit antennas. The conventional precoder $\mathbf{P}$ is dervied by minimizing the minimum square error (MSE)

$$
\epsilon=\mathrm{E}\left[\|\tilde{\mathbf{s}}-\mathbf{s}\|^{2}\right]=\mathrm{E}\left[\left\|\beta^{-1}(\mathbf{H P} \mathbf{s}+\mathbf{n})-\mathbf{s}\right\|^{2}\right],
$$

where $\mathbf{P}$ stands for either $\mathbf{P}_{1}$ or $\mathbf{P}_{2} ; \mathbf{H}$ stands for either $\mathbf{H}_{1}$ or $\mathbf{H}_{2}$. The ZF solution is obtained by minimizing (4) for unconstraint transmit power [14] and can be formed as

$$
\tilde{\mathbf{P}}=\mathbf{H}^{H}\left(\mathbf{H H}^{H}\right)^{-1} \text {. }
$$

Substituting (5) into (1) yields $\mathbf{y}_{r}=\mathbf{s}+\mathbf{n}_{1} / \beta_{1}$. Consequently, for each element of $\mathbf{y}_{r}$, denoted as $y_{i}, i=1, \ldots, B$, we have $y_{i}=s_{i}+n_{i} / \beta_{1}$, where $n_{i} / \beta_{1} \sim \mathcal{C N}\left(0, \sigma_{n_{1}}^{2} / \beta_{1}^{2}\right)$. To enable soft DF, we need to derive soft information for each transmitted symbol in terms of log-likelihood ratio (LLR), which can be computed from the probability density function (PDF) of the received signal at the RS. The PDF of $y_{i}$ conditioned on the transmitted symbol $s_{i}$ can be obtained as

$$
f\left(y_{i} \mid s_{i}=s_{m}\right)=\frac{1}{\sigma_{n_{1}}^{2} / \beta_{1}^{2}} \exp \left(-\frac{\left|y_{i}-s_{m}\right|^{2}}{\sigma_{n_{1}}^{2} / \beta_{1}^{2}}\right)
$$

For BPSK/OQPSK signal, the LLR for $s_{i}$ can be computed as

$$
\begin{aligned}
\lambda\left(s_{i}\right) & =\ln \frac{f\left(y_{i} \mid s_{n}=+1\right)}{f\left(y_{i} \mid s_{n}=-1\right)}=\ln \frac{\exp \left(-\left|y_{i}-1\right|^{2} \beta_{1}^{2} / \sigma_{n_{1}}^{2}\right)}{\exp \left(-\left|y_{i}+1\right|^{2} \beta_{1}^{2} / \sigma_{n_{1}}^{2}\right)} \\
& =\frac{4 \beta_{1}^{2} \operatorname{Re}\left\{y_{i}\right\}}{\sigma_{n_{1}}^{2}}=\frac{4 N_{b} \sigma_{s}^{2} \operatorname{Re}\left\{y_{i}\right\}}{\sigma_{n_{1}}^{2} \operatorname{trace}\left(\tilde{\mathbf{P}}_{1} \tilde{\mathbf{P}}_{1}^{H}\right)}
\end{aligned}
$$

For QPSK/4ASK signals, denoting $s_{i}=x_{i}+j y_{i}$, the LLR value of $x_{i}$ can be computed as

$$
\begin{aligned}
& \lambda\left(x_{i}\right)=\ln \frac{f\left(z_{i} \mid s_{0}\right)+f\left(z_{i} \mid s_{3}\right)}{f\left(z_{i} \mid s_{1}\right)+f\left(z_{i} \mid s_{2}\right)} \approx \ln \frac{\exp \left(-\frac{\left|z_{i}-s_{+}\right|^{2}}{\sigma_{n_{1}}^{2} / \beta_{1}^{2}}\right)}{\exp \left(-\frac{\left|z_{i}-s_{-}\right|^{2}}{\sigma_{n_{1}}^{2} / \beta_{1}^{2}}\right)} \\
& =\frac{\beta_{1}^{2}}{\sigma_{n_{1}}^{2}}\left\{\left|z_{i}-s_{-}\right|^{2}-\left|z_{i}-s_{+}\right|^{2}\right\} \\
& = \begin{cases}\frac{2 \beta_{1}^{2}}{\sigma_{n_{1}}^{2}} \operatorname{Re}\left\{s_{+}^{*} z_{i}-s_{-}^{*} z_{i}\right\} & \text { for QPSK } \\
\frac{\beta_{1}^{2}}{\sigma_{n_{1}}^{2}} \operatorname{Re}\left\{\left[2 s_{+}^{*} z_{i}-\left|s_{+}\right|^{2}\right]-\left[2 s_{-}^{*} z_{i}-\left|s_{-}\right|^{2}\right]\right\} & \text { for 4ASK }\end{cases}
\end{aligned}
$$

where $s_{+}$denotes the QPSK/4ASK symbol corresponding to $\max \left\{f\left(z_{n} \mid s_{0}\right), f\left(z_{n} \mid s_{3}\right)\right\}$, and $s_{-}$denotes the QPSK/4ASK symbol corresponding to $\max \left\{f\left(z_{n} \mid s_{1}\right), f\left(z_{n} \mid s_{2}\right)\right\}$ since the real part of the symbols $s_{0}, s_{3}$ corresponds to 0 , and the real part of the symbols $s_{1}, s_{2}$ corresponds to $1 . \lambda\left(y_{i}\right)$ can be derived similarly.

With the soft DF protocol, the LLRs instead of the hard decisions are passed from the RS to MSs.

\section{B. soft DF protocol with improved precoding}

The conventional solution in (5) is optimum for systems with proper modulations, such as $M$-QAM and $M$-PSK (for which $E\left[\mathbf{s s}^{T}\right]=\mathbf{0}$ ). However, for the improper modulation schemes, such as $M$-ary ASK, OQPSK (for which $E\left[\mathbf{s s}^{T}\right]=$ $\sigma_{s}^{2} \mathbf{I}_{B}$ ), the conventional solution is suboptimum. We propose a new precoding scheme based on an error criterion defined by

$$
\mathbf{e}=\beta^{-1} \operatorname{Re}\{\mathbf{H P s}+\mathbf{n}\}-\mathbf{s},
$$

where $\mathbf{P}$ stands for either $\mathbf{P}_{1}$ or $\mathbf{P}_{2} ; \beta$ stands for either $\beta_{1}$ or $\beta_{2}$. The new precoder results from the following optimization $\mathbf{P}=\arg \min _{\beta, \mathbf{P}} \mathrm{E}\left[\|\mathbf{e}\|^{2}\right]=\arg \min _{\beta, \mathbf{P}} \mathrm{E}\left[\left\|\beta^{-1} \operatorname{Re}\{\mathbf{H P s}+\mathbf{n}\}-\mathbf{s}\right\|^{2}\right]$.

The reason for the above modification is that the conventional optimization approach yields a complex valued filter output. However, only the real part of this output is relevant for the decision in a system with an improper constellation. Thus, minimization of the modified cost function in (9) will result in a better performance [15]. The improved precoder is derived by solving the minimum of the following function with respect to $\mathbf{P}$ and $\beta$

$$
\begin{aligned}
\eta & =\operatorname{trace}\left\{0 . 2 5 \beta ^ { - 2 } \sigma _ { s } ^ { 2 } \left(\mathbf{H} \mathbf{P} \mathbf{P}^{H} \mathbf{H}^{H}+\mathbf{H} \mathbf{P} \mathbf{P}^{T} \mathbf{H}^{T}+\mathbf{H}^{*} \mathbf{P}^{*} \mathbf{P}^{H} \mathbf{H}^{H}\right.\right. \\
& \left.+\mathbf{H}^{*} \mathbf{P}^{*} \mathbf{P}^{T} \mathbf{H}^{T}\right)-0.5 \beta^{-1} \sigma_{s}^{2}\left(\mathbf{H P}+\mathbf{H}^{*} \mathbf{P}^{*}+\mathbf{P}^{H} \mathbf{H}^{H}+\mathbf{P}^{T} \mathbf{H}^{T}\right) \\
& \left.+\left(0.5 \beta^{-2} \sigma_{n_{1}}^{2}+\sigma_{s}^{2}\right) \mathbf{I}\right\} .
\end{aligned}
$$

Using the cyclic property of the trace, we obtain the following:

$$
\begin{aligned}
\frac{\partial \operatorname{trace}\left(\mathbf{H} \mathbf{P} \mathbf{P}^{H} \mathbf{H}^{H}\right)}{\partial \mathbf{P}} & =\frac{\partial \operatorname{trace}\left(\mathbf{P}^{H} \mathbf{H}^{H} \mathbf{H P}\right)}{\partial \mathbf{P}}=\left(\mathbf{H}^{H} \mathbf{H P}\right)^{*} ; \\
\frac{\partial \operatorname{trace}\left(\mathbf{H} \mathbf{P} \mathbf{P}^{T} \mathbf{H}^{T}\right)}{\partial \mathbf{P}} & =\frac{\partial \operatorname{trace}\left(\mathbf{P}^{T} \mathbf{H}^{T} \mathbf{H} \mathbf{P}\right)}{\partial \mathbf{P}}=2 \mathbf{H}^{T} \mathbf{H} \mathbf{P} ; \\
\frac{\partial \operatorname{trace}\left(\mathbf{H}^{*} \mathbf{P}^{*} \mathbf{P}^{H} \mathbf{H}^{H}\right)}{\partial \mathbf{P}} & =\frac{\partial \operatorname{trace}\left(\mathbf{P}^{H} \mathbf{H}^{H} \mathbf{H}^{*} \mathbf{P}^{*}\right)}{\partial \mathbf{P}}=\mathbf{0} ; \\
\frac{\partial \operatorname{trace}\left(\mathbf{H}^{*} \mathbf{P}^{*} \mathbf{P}^{T} \mathbf{H}^{T}\right)}{\partial \mathbf{P}} & =\frac{\partial \operatorname{trace}\left(\mathbf{P}^{T} \mathbf{H}^{T} \mathbf{H}^{*} \mathbf{P}^{*}\right)}{\partial \mathbf{P}}=\mathbf{H}^{T} \mathbf{H}^{*} \mathbf{P}^{*} .
\end{aligned}
$$

Furthermore, we also obtain the following partial derivatives

$$
\begin{aligned}
& \frac{\partial \operatorname{trace}(\mathbf{H P})}{\partial \mathbf{P}}=\mathbf{H}^{T} ; \quad \frac{\partial \operatorname{trace}\left(\mathbf{P}^{T} \mathbf{H}^{T}\right)}{\partial \mathbf{P}}=\mathbf{H}^{T} ; \\
& \frac{\partial \operatorname{trace}\left(\mathbf{H}^{*} \mathbf{P}^{*}\right)}{\partial \mathbf{P}}=\frac{\partial \operatorname{trace}\left(\mathbf{P}^{H} \mathbf{H}^{H}\right)}{\partial \mathbf{P}}=\mathbf{0} .
\end{aligned}
$$

Setting $\frac{\partial \eta}{\partial \mathbf{P}}=\mathbf{0}$, and using (12), (13) yields

$$
\begin{gathered}
0.25 \beta^{-2} \sigma_{s}^{2}\left[\left(\mathbf{H}^{H} \mathbf{H} \mathbf{P}\right)^{*}+2 \mathbf{H}^{T} \mathbf{H} \mathbf{P}+\mathbf{H}^{T} \mathbf{H}^{*} \mathbf{P}^{*}\right] \\
-0.5 \beta^{-1} \sigma_{s}^{2}\left(2 \mathbf{H}^{T}\right)=\mathbf{0}
\end{gathered}
$$

Subsequently, we obtain

$$
\mathbf{H}^{H} \mathbf{H P}+\mathbf{H}^{H} \mathbf{H}^{*} \mathbf{P}^{*}=2 \beta \mathbf{H}^{H},
$$


which is equivalent to

$$
\mathbf{H}^{H} \mathbf{H} \tilde{\mathbf{P}}+\mathbf{H}^{H} \mathbf{H}^{*} \tilde{\mathbf{P}}^{*}=2 \mathbf{H}^{H},
$$

where $\tilde{\mathbf{P}}=\mathbf{P} / \beta$. Let us denote $\tilde{\mathbf{P}}=\tilde{\mathbf{P}}_{r}+j \tilde{\mathbf{P}}_{i}, \mathbf{H}^{H} \mathbf{H}=$ $\mathbb{H}_{r}+j \mathbb{H}_{i}, \quad \mathbf{H}^{H} \mathbf{H}^{*}=\mathbb{H}_{r}^{\prime}+j \mathbb{H}_{i}^{\prime}, \quad 2 \mathbf{H}^{H}=2 \mathbf{H}_{r}^{H}+j 2 \mathbf{H}_{i}^{H}$. Equation (14) can be reformed as

$$
\begin{aligned}
2 \mathbf{H}_{r}^{H}+j 2 \mathbf{H}_{i}^{H} & =\left(\mathbb{H}_{r}+j \mathbb{H}_{i}\right)\left(\tilde{\mathbf{P}}_{r}+j \tilde{\mathbf{P}}_{i}\right) \\
& +\left(\mathbb{H}_{r}^{\prime}+j \mathbb{H}_{i}^{\prime}\right)\left(\tilde{\mathbf{P}}_{r}-j \tilde{\mathbf{P}}_{i}\right) \\
& =\left(\mathbb{H}_{r} \tilde{\mathbf{P}}_{r}-\mathbb{H}_{i} \tilde{\mathbf{P}}_{i}+\mathbb{H}_{r}^{\prime} \tilde{\mathbf{P}}_{r}+\mathbb{H}_{i}^{\prime} \tilde{\mathbf{P}}_{i}\right) \\
& +j\left(\mathbb{H}_{r} \tilde{\mathbf{P}}_{i}+\mathbb{H}_{i} \tilde{\mathbf{P}}_{r}+\mathbb{H}_{i}^{\prime} \tilde{\mathbf{P}}_{r}-\mathbb{H}_{r}^{\prime} \tilde{\mathbf{P}}_{i}\right) .
\end{aligned}
$$

Then $2 \mathbf{H}_{r}^{H}$ and $2 \mathbf{H}_{i}^{H}$ can be expressed in vector form as

$$
\left[\begin{array}{l}
2 \mathbf{H}_{r}^{H} \\
2 \mathbf{H}_{i}^{H}
\end{array}\right]=\left[\begin{array}{ll}
\mathbb{H}_{r}+\mathbb{H}_{r}^{\prime} & \mathbb{H}_{i}^{\prime}-\mathbb{H}_{i} \\
\mathbb{H}_{i}+\mathbb{H}_{i}^{\prime} & \mathbb{H}_{r}-\mathbb{H}_{r}^{\prime}
\end{array}\right]\left[\begin{array}{c}
\tilde{\mathbf{P}}_{r} \\
\tilde{\mathbf{P}}_{i}
\end{array}\right],
$$

which leads to the improved ZF solution $\mathbf{P}=\beta \tilde{\mathbf{P}}=\beta \tilde{\mathbf{P}}_{r}+$ $j \beta \tilde{\mathbf{P}}_{i}$, where $\tilde{\mathbf{P}}_{r}$ and $\tilde{\mathbf{P}}_{i}$ are derived as

$$
\left[\begin{array}{c}
\tilde{\mathbf{P}}_{r} \\
\tilde{\mathbf{P}}_{i}
\end{array}\right]=\left[\begin{array}{ll}
\mathbb{H}_{r}+\mathbb{H}_{r}^{\prime} & \mathbb{H}_{i}^{\prime}-\mathbb{H}_{i} \\
\mathbb{H}_{i}+\mathbb{H}_{i}^{\prime} & \mathbb{H}_{r}-\mathbb{H}_{r}^{\prime}
\end{array}\right]^{-1}\left[\begin{array}{l}
2 \mathbf{H}_{r}^{H} \\
2 \mathbf{H}_{i}^{H}
\end{array}\right]
$$

With the improved precoder, the received signal at the RS can be expressed as

$$
\mathbf{y}_{r}^{\prime}=\operatorname{Re}\left(\mathbf{H}_{1} \tilde{\mathbf{P}}_{1}^{\prime} \mathbf{s}+\mathbf{n}_{1} / \beta_{1}^{\prime}\right)=\mathbf{s}+\operatorname{Re}\left(\mathbf{n}_{1}\right) / \beta_{1}^{\prime},
$$

where the parameter $\beta_{1}^{\prime}$ is calculated in the same way as shown in (2). Consequently, for each element of $\mathbf{y}_{r}^{\prime}$, denoted as $y_{i}^{\prime}, i=1, \ldots, B$, we have $y_{i}^{\prime}=s_{i}+w_{i}$, where $w_{i} \sim \mathcal{N}\left(0, \sigma_{n}^{2} /\left(2 \beta_{1}^{\prime 2}\right)\right)$. The PDF of $y_{i}^{\prime}$ conditioned on the transmitted symbol $s_{i}$ can be obtained as

$$
f\left(y_{i}^{\prime} \mid s_{i}=s_{m}\right)=\frac{1}{2 \sigma_{n_{1}}^{2} / \beta_{1}^{\prime 2}} \exp \left(-\frac{\left|y_{i}^{\prime}-s_{m}\right|^{2}}{2 \sigma_{n_{1}}^{2} / \beta_{1}^{\prime 2}}\right)
$$

The LLR for the BPSK/OQPSK symbol $s_{i}$ can be computed as

$$
\begin{aligned}
\lambda\left(s_{i}\right) & =\ln \frac{f\left(y_{i}^{\prime} \mid s_{n}=+1\right)}{f\left(y_{i}^{\prime} \mid s_{n}=-1\right)}=\ln \frac{\exp \left(-\left(y_{i}^{\prime}-1\right)^{2} \beta_{1}^{\prime 2} / 2 \sigma_{n_{1}}^{2}\right)}{\exp \left(-\left(y_{i}^{\prime}+1\right)^{2} \beta_{1}^{\prime 2} / 2 \sigma_{n_{1}}^{2}\right)} \\
& =\frac{2 \beta_{1}^{\prime 2} y_{i}^{\prime}}{\sigma_{n_{1}}^{2}}=\frac{2 N_{b} \sigma_{s}^{2} y_{i}^{\prime}}{\sigma_{n_{1}}^{2} \operatorname{trace}\left(\tilde{\mathbf{P}}_{1}^{\prime} \tilde{\mathbf{P}}_{1}^{\prime, H}\right)}
\end{aligned}
$$

The LLR for the QPSK/4ASK signals can be derived similarly.

\section{NumericAl RESUlts}

Simulation results are presented in this section to demonstrate the effectiveness of the proposed soft DF protocol and linear processing schemes. Assume the BS has 4 transmit antennas and the RS also has 4 transmit and receive antennas $\left(N_{b}=N_{r}=4\right)$. The transmit power is set to $N_{b} \sigma_{s}^{2}=4$, i.e., unit average transmit power is used for each transmitted symbol. We assume uncorrelated Rayleigh fading channel. The simulation results are averaged over at least 50,000 channel realizations. Direct implementation of (16) leads to the numerical problem caused by the inversion of an ill-conditioned matrix. However, the problem can be easily resolved by regularizing the inverse [16]. the regularization factor is chosen to be a very small number.
Precoded Fixed Relay Networks with AF, DF and SDF

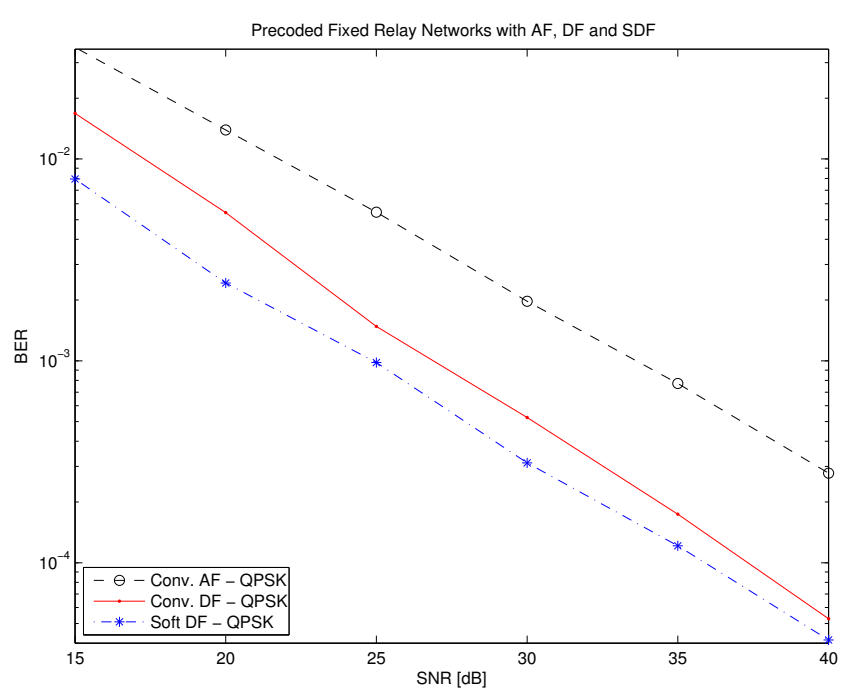

Fig. 2. Performance comparison between the conventional AF, DF and the proposed soft DF systems, the conventional precoding scheme is used.

First, we compare different relaying protocols with the conventional precoding scheme in Fig. 2. One can see that the DF scheme significantly outperform the AF scheme in the studied 2-hop fixed relay network, the gap can be up to $7 \mathrm{~dB}$. Also considering the fact that the additional complexity (compared to AF protocol) is only spent at the RS, rather than in the MSs, it is preferable to employ the DF protocol in fixed relay networks. Fig. 2 also shows that performance improvement can be obtained by passing soft information (instead of hard decisions) in terms of LLRs from relay to mobile users. The gain achieved by the proposed soft DF protocol over the conventional DF protocol is more obvious at low SNRs due to the fact that the conventional hard decision based DF scheme is more vulnerable to error propagation at low SNRs.

Fig. 3 shows the comparison between the conventional precoding scheme and the proposed precoding scheme in AF based fixed relay networks. The employed modulation schemes are 4ASK, QPSK, OQPSK, which are chosen such that all the systems have the same data transmission rate or spectrum efficiency. In both 4ASK and QPSK/OQPSK systems, the gain by applying the improved precoding can be up to $5 \mathrm{~dB}$.

Different variants of the DF schemes are compared in Fig. 4. It can be observed from the figure that 1) with conventional DF protocol and linear precoding scheme, the QPSK system outperforms the 4ASK system; 2) the 4ASK/OQPSK system with the improved linear precoding performs much better than the 4ASK/QPSK system with the conventional linear precoding; 3) the best performance is achieved by the OQPSK system with the soft DF protocol coupled with the improved linear precoding, e.g., it outperforms the OQPSK system with the conventional DF protocol with the improved linear precoding by $1.4 \mathrm{~dB}$ and outperforms the 4ASK system with the improved linear precoding by $5 \mathrm{~dB}$; 4) comparing Fig. 4 with Fig. 3, one can clearly see that it is more advantageous to apply the improved linear precoding in the DF based relay 
Precoded Fixed Relay Networks with AF

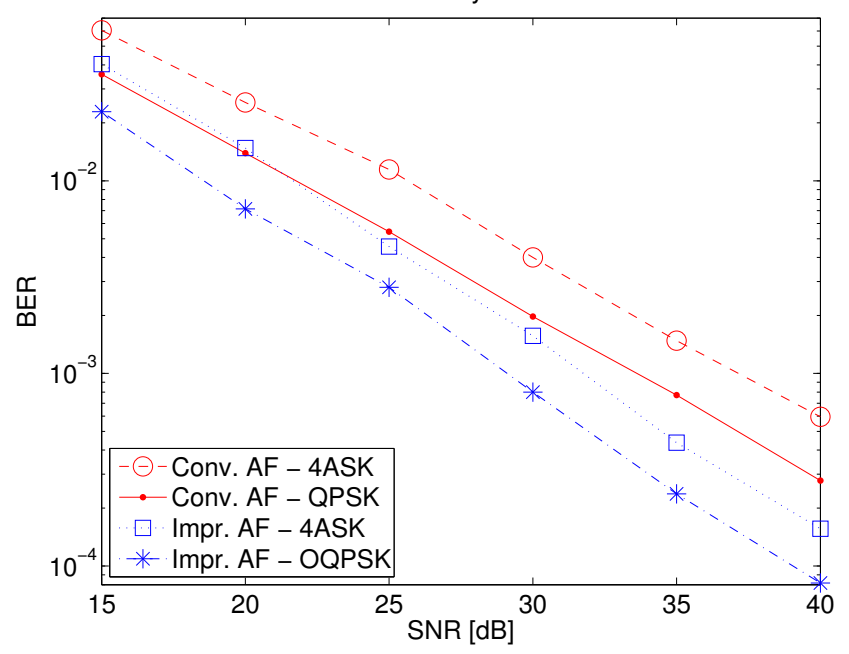

Fig. 3. Performance comparison between conventional precoding and proposed precoding in AF based fixed relay networks.

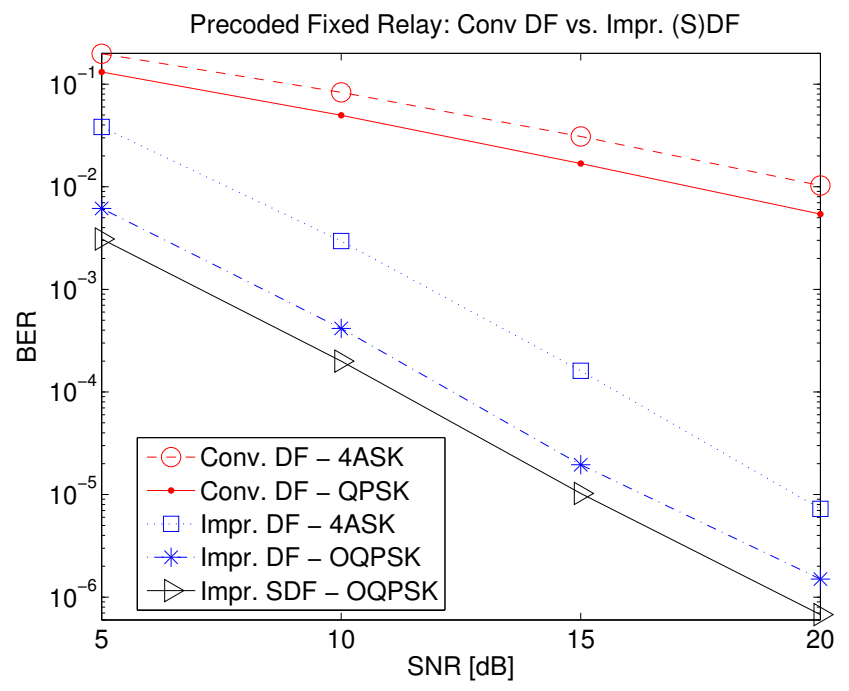

Fig. 4. Performance comparison between different DF based systems.

systems rather than in the AF based systems. It further justifies the use of DF and soft DF protocol in fixed relay networks.

The computation of the improved linear precoding matrix is slightly more complex than the computation of the conventional linear precoding matrix due to inversion of a dimension doubled matrix (it is obvious to see by comparing Equ. (5) and (16)). However, once the precoding matrices are derived, the improved and the conventional systems have exactly the same pre-processing complexity in the transmitter. Therefore, the complexity increase by the improved schemes is not significant compared to the conventional ones, especially in slow-fading channels for which the precoding matrices do not need to be updated frequently. Also considering the fact that the precoding operations are conducted at the base station and relay station which possess strong signal processing capabilities, the nonsignificant complexity increase is thus affordable and is also largely compensated by the significant performance

\section{Conclusions}

In this paper, we consider multiuser relay channel to support data transmission from a source to multiple destinations via a relay station, while most prior work on relay channel focuses on the standard channel where relays are used to help send data from a single source to a single destination. We mainly considered DF relays, and proposed a soft-decode-and-forward protocol to mitigate the error propagation problem inherent in the conventional DF systems. We also proposed an advanced linear processing strategy which provides significant performance improvement over the conventional linear processing, and the performance gain is much greater in the DF systems (especially in the proposed soft DF systems) than in the AF systems.

\section{REFERENCES}

[1] R. Pabst, B. Walke, D. Schultz, D. Herbhold, H. Yanikomeroglu, S. Mukherjee, H. Viswanathan, W. Zirwas, M. Dohler, H. Aghvami, D. Falconer, G. Fettweis. "Relay-based deployment concepts for wireless and mobile broadband radio," IEEE Commun. Mag., vol. 42, no. 9, pp. 80-89, Sept. 2004.

[2] J. Laneman, D. Tse, G. Wornell. "Cooperative diversity in wireless networks: efficient protocols and outage behavior," IEEE Trans. on Inform. Theory, vol. 50, no. 12, pp. 3062-3080, Dec. 2004.

[3] R. Nabar, H. Bolcskei, F. Kneubuhler, "Fading relay channels: performance limits and space-time signal design," IEEE J. Select. Areas Commun., pp. 10991109, Aug. 2004.

[4] J. Laneman and G. Wornell. "Distributed space-time-coded protocols for exploiting cooperative diversity in wireless network," IEEE. Trans. Inform. Theory, vol. 49, pp. 24152425, Oct. 2003.

[5] Y. Jing, B. Hassibi. "Distributed space-time coding in wireless relay networks," IEEE Trans. Wireless Commun., vol. 5, no. 12, pp. 35243536, Dec. 2006.

[6] Q. Spencer, C. Peel, A. Swindlehurst, M. Haardt. "An introduction to the multi-user MIMO downlink," IEEE Commun. Mag., vol. 42, no. 10, pp. 60-67, Oct. 2004.

[7] A. Sendonaris, E. Erkip, and B. Aazhang. "User cooperation diversity part I: System description,” IEEE Trans. Commun., vol. 51, pp. 19271938, Nov. 2003.

[8] J. Yu, D. Liu, C. Yin, G. Yue. "Relay-assisted MIMO multiuser precoding in fixed relay networks," Proc. International Conference on Wireless Communications, Networking and Mobile Computing, pp. 881-884, Sept. 2007.

[9] B. Yoo, K. Lee, H. Kim, C. Lee. "Linear precoding techniques for 2-Hop MIMO relaying”. Proc. ITC-CSCC'08, pp. 1761-1764.

[10] C-B. Chae, T. Tang, R. Health, S. Cho. "MIMO relaying with linear processing for multiuser transmission in fixed relay networks," IEEE Trans. Sig. Proc., vol. 56, no. 2, pp. 727-738, Feb. 2008.

[11] T. Tang, C. Chae, R. Heath, S. Cho. "On achievable sum rates of a multiuser MIMO relay channel," Proc. IEEE ISIT, pp. 1026-1030, July 2006.

[12] R. Hoshyar, R. Tafazolli. "Soft decode and forward of MQAM modulations for cooperative relay channels," Proc. IEEE VTC'08 Spring, pp. 639-643, May 2008.

[13] H. Sneessens, L. Vandendorpe. "Soft decode and forward improves cooperative communications," Proc. IEE International Conference on $3 G$ and Beyond, pp. 73-76, Nov. 2005.

[14] B. Vojcic, W. Jang. "Transmitter precoding in synchronous multiuser communication", IEEE Trans. Commun., vol. 46, no. 10, pp. 1346-1355, Oct. 1998

[15] P. Xiao, R. Carrasco, I. Wassell. "Generalized equalization algorithm utilizing improper ISI", IEEE Trans. on on Veh. Technol., vol. 58, no. 2, pp. 788-799, Feb. 2009.

[16] B. Hochwald, C. Peel, A. Swindlehurst. "A vector-perturbation technique for near-capacity multiantenna multiuser communication - part I: channel inversion and regularization", IEEE Trans. Commun., vol 53, no. 1, pp. 195-202, Jan 2005 